\title{
The Impact of Reliability Evaluation on a Semi-supervised Learning Approach
}

\author{
Pasquale Foggia $^{1}$, Gennaro Percannella ${ }^{1}$, Carlo Sansone $^{2}$, and Mario Vento ${ }^{1}$ \\ 1 Dipartimento di Ingegneria dell'Informazione e di Ingegneria Elettrica, \\ Università di Salerno, Via Ponte Don Melillo, 1 I-84084 Fisciano (SA), Italy \\ \{pfoggia, pergen, mvento\}@unisa.it \\ 2 Dipartimento di Informatica e Sistemistica, \\ Università degli Studi di Napoli Federico II, Via Claudio, 21 I-80125 Napoli, Italy \\ carlosan@unina.it
}

\begin{abstract}
In self-training methods, unlabeled samples are first assigned a provisional label by the classifier, and then used to extend the training set of the classifier itself. For this latter step it is important to choose only the samples whose classification is likely to be correct, according to a suitably defined reliability measure.

In this paper we want to study to what extent the choice of a particular technique for evaluating the classification reliability can affect the learning performance. To this aim, we have compared five different reliability evaluators on four publicly available datasets, analyzing and discussing the obtained results.
\end{abstract}

\section{Introduction}

In the last years the Pattern Recognition community tried to use semi-supervised learning [211] to answer the needs of those real-life problems in which labeled data for training a classifier are usually a small proportion of the total available data 9]. This can happen when it is very difficult and time-consuming to obtain labeled objects 610 or when labeling is dangerous or even destructive [9].

A possible approach for building a better classifier by using large amount of unlabeled data together with the labeled data is in fact semi-supervised learning, that is "halfway between supervised and unsupervised learning" [2]. Among the various technique for semi-supervised learning, self-training is probably the earliest and also the most commonly used one [11], since it does not require any particular assumption. The approach that is typically followed by self-training methods is to augment the labeled training set of a classifier by using also those unlabeled samples that it classifies with the highest reliability 461011. In practice, the classifier is first trained with labeled data. Then, it is used to classify unlabeled data; the most confident unlabeled samples, together with their predicted labels, are added to the training set. This procedure is iterated until a stopping criterion is satisfied.

However, a problem that has been disregarded until now is how to choose a suitable approach for evaluating the classification reliability of unlabeled samples. In most papers [410] the authors simply suggest to select samples classified 
with the highest confidence, even if there are several methods proposed so far for evaluating classification reliability (see for example 358]). A correct choice of the approach used for evaluating classification reliability should be instead of particular interest in the semi-supervised learning, since a classifier can also deteriorate when trained with unlabeled data. In fact, while some researchers demonstrate theoretically that, if the underlying model is guessed correctly, unlabeled data are expected to improve on the error [11, the use of unlabeled data may be more a damage than a benefit if there is a modeling error [9].

Starting from the previous considerations, in this paper we want to evaluate the impact of the choice of a proper reliability evaluation technique in the context of a particular semi-supervised approach, namely the self-training one. Moreover, we also tried to individuate some guidelines that could be used for individuating when a self-training procedure should produce a beneficial impact on the classifier performance. In order to do that, we compared on four publicly available datasets the performance of five different reliability evaluation techniques as the number of the labeled samples varies.

The rest of the paper is organized as follows: in Section 2, the considered self-training procedure is presented. Then, in Section 3 the selected methods for evaluating classification reliability are discussed in details, while the experimental comparative evaluation on some publicly available databases is reported in Section 4. Finally, some concluding remarks are drawn.

\section{The Considered Semi-supervised Method}

As we mentioned in the introduction, we will consider in this work the impact of reliability evaluation on a self-training semi-supervised learning algorithm. In Figure 1, an architectural overview of this algorithm is depicted.

In such a system, we have a classifier that is initially trained with the available labeled samples, and is used to classify the larger amount of unlabeled samples. The output of the classifier is fed into a Reliability Evaluation block, which estimates the reliability of each classification result, giving a numeric reliability value in the range between 0 (completely unreliable) and 1 (completely reliable). Then a Thresholding block divides the samples on the basis of a suitably tuned threshold $\tau$ on the reliability estimate. The ones with a reliability above the threshold are added to the training set, and the classifier is retrained. At this point the ones with a reliability below the threshold are processed again.

These steps are usually repeated until the system finds no more samples that can be added to the training set, although more complex termination conditions could be applied. After the termination of the algorithm, the trained classifier is ready for its use.

Any trainable classifier can be used with this algorithm, provided that a suitable reliability estimator can be defined. Most reliability estimation techniques, however, assume that the classifier delivers a vector of measures related to the likelihood of each class (type 3 classifiers). Since we are interested in assessing the impact of the reliability evaluation on the performance of self-training 


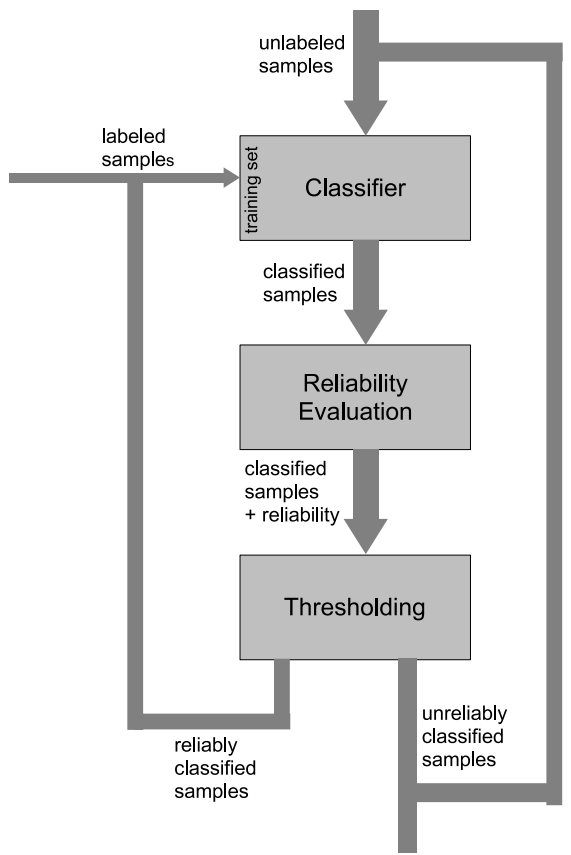

Fig. 1. Architecture of a self-training system based on reliability evaluation

algorithms, we will consider the simplest type 3 classifier, that is the Nearest Neighbor (NN) classifier.

In the following section, we will describe the reliability estimation methods that we have considered for comparison.

\section{The Reliability Evaluation Methods}

For the Reliability Evaluation block, we have chosen three approaches that differ on the amount of training needed to perform the evaluation of the reliability. The considered options are: $(i)$ no training (besides the setup of a global parameter), as represented by the $\psi_{A}$ and the $\psi_{\text {med }}$ estimators; ( $\left.i i\right)$ use of a reference set, as exemplified by the Local_Distance-based_pre and Local_Distance-based_post estimators; (iii) use of a trainable function estimator, as represented by the $\epsilon$-SVR estimators.

The following subsections will provide more details on each method. Notice that except for $\psi_{A}$ and $\psi_{\text {med }}$, the definition of the considered reliability estimators is independent of the adopted classifier architecture. In the descriptions we will use the term reference set to denote the set of labeled samples available to the classifier, following the terminology commonly adopted for NN classifiers; however this does not imply that a different classification algorithm cannot be used. 


\subsection{The $\psi_{A}$ and $\psi_{m e d}$ Estimators}

The first considered approach, introduced in [3], is based on the observation that there are two possible causes for unreliability in a classification: $(i)$ the sample being classified is too different from samples the classifier has seen in the training phase; $(i i)$ the sample being classified lies on the boundary between two classes. Thus, the authors propose for different kinds of classifiers two measures, $\psi_{A}$ and $\psi_{B}$, that are related to the two types of unreliability. In particular, for a NN classifier, the measures are:

$$
\psi_{A}=\max \left\{1-\frac{O_{\text {win }}}{D_{\max }}, 0\right\} ; \psi_{B}=1-\frac{O_{\text {win }}}{O_{2 \text { win }}}
$$

where $O_{\text {win }}$ is the output of the classifier for the winner class, i.e. the distance of the sample from its nearest neighbor in the reference set; $O_{2 \text { win }}$ is the output of the classifier for the second best class, i.e. the distance of the sample from the nearest neighbor belonging to a different class than the absolute nearest neighbor; and $D_{\max }$ is the maximum distance between a sample in the reference set and its nearest neighbor.

It can be easily seen that $\psi_{A}$ takes into account the first cause of unreliability, since it is 1 if the classified sample is in the reference set, and becomes 0 as the sample gets farther from the reference set. Similarly, $\psi_{B}$ reflects the second cause of unreliability, since it is 0 when the classified sample lies at the midpoint between two reference samples of different classes, and tends to 1 when the sample gets unambiguously closer to a single reference sample.

The authors of [3] suggests different ways of combining $\psi_{A}$ and $\psi_{B}$ to obtain a single, comprehensive estimator. For our evaluation we have chosen:

$$
\psi_{\text {med }}=\frac{\psi_{A}+\psi_{B}}{2}
$$

together with the use of $\psi_{A}$ alone.

\subsection{The Local_Distance-Based_Pre and Local_Distance-Based_Post Estimators}

The second approach, introduced in [7], is based on an extension of the $k$ - $N N$ classification algorithm, combined with the leave-one-out technique used for the validation of classifiers. The underlying idea is that the samples in the reference set can be used to estimate the classifier reliability in the region surrounding them in the feature space (hence the term "local" in the name), on the basis of the correctness of their classification performed according to the leave-one-out method.

Before describing the estimators, we need to introduce some notational conventions. We indicate with $R=\left\{r_{1}, \ldots, r_{n}\right\}$ the reference set, and with class $\left(r_{i}\right)$ the true class of reference sample $r_{i}$. We denote as $l\left(r_{i}\right)$ the class that would be 
attributed to sample $r_{i}$ by the classifier using $R-r_{i}$ as its reference set (this is the leave-one-out approach). Then we introduce the correct classification indicator $p$ as the function:

$$
p: r \in R \longrightarrow \begin{cases}1 & \text { if } l(r)=\operatorname{class}(r) \\ 0 & \text { if } l(r) \neq \operatorname{class}(r)\end{cases}
$$

Now, we can define the first reliability estimator, Local_Distance-based_pre (LDpre for short). Given the sample $x$ under examination, we consider the set $N^{k}(x)$ formed by the $k$ nearest neighbors to $x$ in $R$. We have:

$$
\text { LDpre }=\frac{\sum_{r \in N^{k}(x)} p(r) / d(x, r)}{\sum_{r \in N^{k}(x)} 1 / d(x, r)}
$$

where $d(x, r)$ is the distance in feature space (according to a suitably chosen metric) between the classified sample $x$ and the reference sample $r$. In other words, LDpre is a weighted average of the correct classification indicator over the $k$ nearest neighbors to $x$, using as weights the inverse of the distances.

The LDpre estimator does not take into account the class attributed to the classified sample $x$ (hence the "pre" suffix in the name). In contrast, the Local_Distance-based_post estimator (LDpost for short) uses also this information in the reliability evaluation. Namely, instead of averaging the correct classification indicator over the $k$ nearest neighbors in $R$, it considers the $k$ nearest neighbors in the set $\{r \in R: l(r)=l(x)\}$, where, with a slight notational inconsistency, we denote by $l(x)$ the class attributed by the complete classifier to the sample $x$ (recall that $l(r)$ is instead the class attributed to $r$ by a leave-one-out approach).

In other words, the LDpost estimator only considers the reference samples that the leave-one-out classifier has attributed to the same class to which the complete classifier is assigning $x$. If we denote the $k$ nearest neighbors in this subset of $R$ as $N^{k}(x, l(x))$, then we can define LDpost as:

$$
\text { LDpost }=\frac{\sum_{r \in N^{k}(x, l(x))} p(r) / d(x, r)}{\sum_{r \in N^{k}(x, l(x))} 1 / d(x, r)}
$$

\subsection{The $\epsilon$-SVR Estimator}

The third reliability evaluation approach has been proposed in [5]. The basic idea in this approach is to use a trainable function estimator to learn the relation between the reliability and the region in classifier input/output space which the sample belongs to. In particular, the $\epsilon-S V R$ regression algorithm is used as the trainable function estimator. 
After a learning phase, the $\epsilon$-SVR receives as its input the feature vector concatenated with the response of the classifier (encoded as a vector of binary values), and produces as its output the reliability value.

In order to use as the reliability estimator a supervised learning algorithm such as the $\epsilon$-SVR, there are two problems that must be faced: $(i)$ the reliability estimator must be trained using a validation set containing both samples that are classified reliably and samples classified unreliably; the validation set must be different from the reference set, or else (at least for the NN classifier) it would contain only perfectly classified samples; ( $i i)$ for each sample in the validation set, a desired reliability value must be provided during the training of the reliability estimator; this value should reflect as close as possible the actual classification reliability of the sample.

The first problem is particularly hard in our context, since we assume that labeled samples are scarce. So we have adopted for training the $\epsilon$-SVR a technique that is based on $k$-fold cross-validation. In particular, the reference set is randomly partitioned into $F$ disjoint subsets $V_{1}, \ldots, V_{F}$ (called folds) of equal size. The samples of each $V_{i}$ are then classified using $R-V_{i}$ as the reference set; for analogy with the previous subsection, we will denote as $l(v)$ the class assigned to sample $v \in V_{i}$ in this step, and as $p(v)$ the correct classification indicator, that is 1 if $l(v)=\operatorname{class}(v)$, and 0 otherwise. At this point we can use all the samples of the reference set, together with the class labels $l(v)$ assigned by the folding procedure, for the training phase of the $\epsilon$-SVR, since we expect that they will not be all correctly classified.

For the second problem (the definition of the desired reliability values), we have used $p(v)$ as the desired reliability value for sample $v$. While $p(v)$ only assumes the values 0 and 1 , the generalization ability of the $\epsilon$-SVR manages to interpolate other values inside the $[0,1]$ interval.

\section{Experimental Results}

The questions raised in this paper can be summarized as follows: $(i)$ what is the impact of the use of different reliability estimators on the recognition performance when the self-training procedure is used? $(i i)$ how the performance of the self-training depend on the size of the training set?

In this section, we provide an answer to the above questions from an experimental point of view. In the design of the experimental protocol several aspects have been taken into account: the architecture of the base classifier, the settings of the reliability estimators, the criterion to stop the self-training procedure, the size of the training set, the value of the reliability threshold. The way these aspects have been taken into account in the tests is summarized in the following.

Base classifier: tests were carried out by using a Nearest Neighbor (NN). This choice is motivated by the fact that the NN does not require a training procedure, and is the simplest type 3 classifier. 
Reliability estimators: several preliminary tests have been carried out to determine the best setup of the LDpre, LDpost and $\epsilon$-SVR reliability estimators: for the first two estimators the analysis has been carried out with respect to the number $k$ of nearest neighbors, while for the latter we searched for the optimal number $F$ of the folds the reference set is partitioned into. The results reported afterwards consider only the best configurations. The $\psi_{A}$ and $\psi_{m e d}$ did not require a setup procedure.

Self-training stopping criterion: we stop the self-training procedure when no more samples are available that can be added to the training set. This happens when the reliability of all the classified samples is below the threshold $\tau$. However, our tests accounted also for the performance obtained by using an ideal stopping criterion, i.e. by stopping the self-training procedure when the minimum error rate is reached. This analysis is useful to highlight the potentiality of self-training using a certain reliability estimator, as it represents the highest performance that can be obtained. For simplicity, hereinafter we will refer to these stopping criteria as END and BEST respectively.

Training set size: we analyzed how recognition performance vary with the size of the dataset when using self-training with different reliability estimators. We considered several configurations where the reference set used by the NN ranges within $2 \%$ to $15 \%$ of the whole dataset.

Table 1. Characteristics of the employed datasets

\begin{tabular}{|c||cccc|}
\hline \hline Data set & letter & pendigits & spam & thyroid \\
\hline \hline \# classes & 26 & 10 & 2 & 3 \\
\hline \# features & 16 & 16 & 57 & 21 \\
\hline \# patterns & 20000 & 10992 & 4601 & 7200 \\
\hline \# patterns used for test & 2000 & 1100 & 460 & 3428 \\
\hline \hline
\end{tabular}

Table 2. Error rates obtained on the considered datasets by the base NN and by the NN after the self-training procedure using the different methods for reliability estimation. Performance improvements and decreases with respect to the base $\mathrm{NN}$ are reported with a plus (+) and minus (-) sign, respectively. The best result for each dataset is highlighted in bold.

\begin{tabular}{|c||c|c||c|c||c|c||c|c|}
\hline \hline & $\tau$ & letter & $\tau$ & pendigits & $\tau$ & spam & $\tau$ & thyroid \\
\hline \hline Base NN & - & $35.2 \%$ & - & $7.64 \%$ & - & $19.13 \%$ & - & $9.48 \%$ \\
\hline \hline$\psi_{A}$ & 0.90 & $\mathbf{2 9 . 5 0} \%(+)$ & 0.85 & $\mathbf{5 . 6 4} \%(+)$ & 0.90 & $22.39 \%(-)$ & 0.95 & $9.31 \%(+)$ \\
\hline$\psi_{\text {med }}$ & 0.85 & $30.70 \%(+)$ & 0.80 & $5.82 \%(+)$ & 0.90 & $20.87 \%(-)$ & 0.95 & $\mathbf{8 . 6 6} \%(+)$ \\
\hline LD-pre & 0.27 & $34.75 \%(+)$ & 0.70 & $8.37 \%(-)$ & 0.85 & $20.65 \%(+)$ & 0.55 & $8.91 \%(+)$ \\
\hline LD-post & 0.27 & $57.30 \%(-)$ & 0.70 & $28.36 \%(-)$ & 0.75 & $\mathbf{1 8 . 9 1} \%(+)$ & 0.60 & $9.80 \%(-)$ \\
\hline$\epsilon-$ SVR & 0.90 & $32.60 \%(+)$ & 0.85 & $7.37 \%(+)$ & 0.85 & $21.74 \%(-)$ & 0.65 & $9.16 \%(+)$ \\
\hline \hline
\end{tabular}




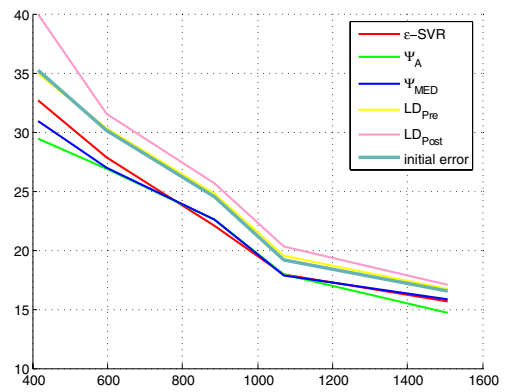

(a)

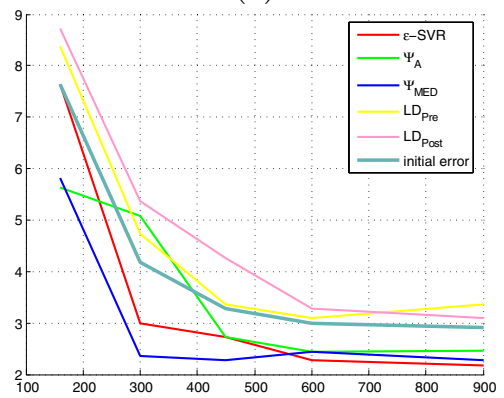

(c)

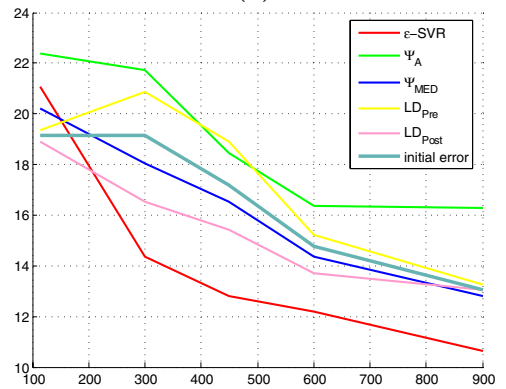

(e)

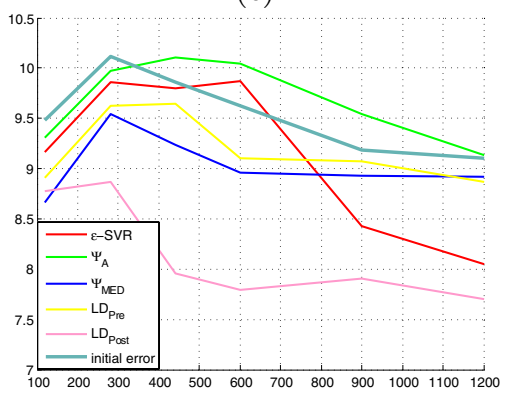

(g)

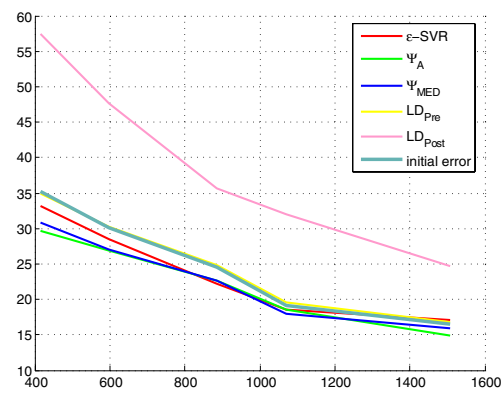

(b)

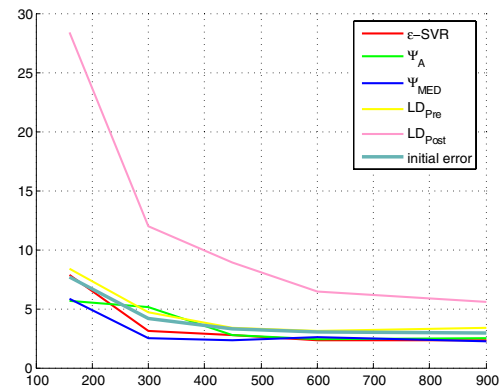

(d)

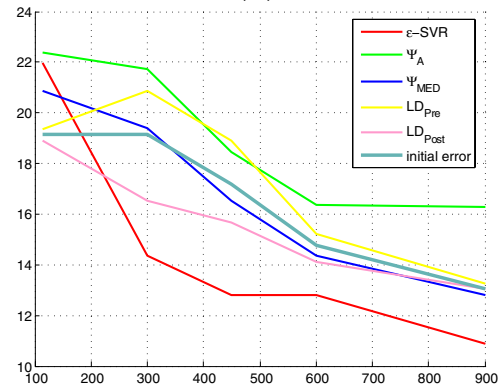

(f)

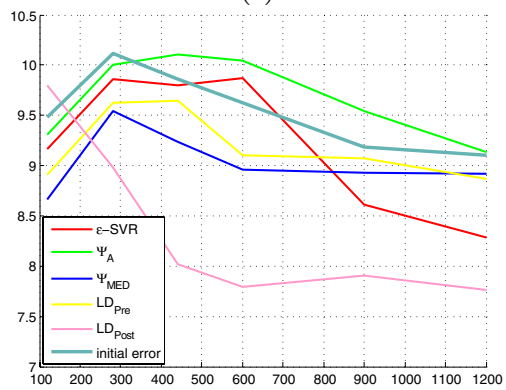

(h)

Fig. 2. Trends of error rate with respect to the size of the reference set used for the $\mathrm{NN}$ in case of (a),(b) letter, (c),(d) pendigits, (e),(f) spam, (g),(h) thyroid datasets. Curves in the left/right column are obtained using the BEST/END criterion. 
Reliability threshold $\tau$ : for each reliability estimator we selected the value of the threshold $\tau$ that optimized the recognition performance when the reference set used by the $\mathrm{NN}$ is $2 \%$ of the whole dataset. Then, these optimal values were used for all the remaining experimentations.

We have considered four different datasets coming from the UCI Machine Learning Repository [1, i.e. letter, pendigits, spam and thyroid. Their characteristics are summarized in Table 1, where we have reported the number of the classes, of the features and of the samples within the whole dataset and those used for the test.

The results of the first experiment are reported in Table2. Here, the error rates obtained by the NN after the self-training procedure using the different methods for reliability estimation on the considered datasets are reported together with the performance of the base classifier without semi-supervised training. The results refers to the case where the reference set used by the $\mathrm{NN}$ is $2 \%$ of the dataset (as in 9]). For each configuration of the self-training procedure and for each dataset the results shown in Table 21 represent the best performance with respect to the threshold $\tau$ defined in Section 2, whose optimal values are reported in the same Table.

The results in Table 2 clearly show that the use of the self-training procedure is beneficial on all the datasets: in all cases we observe the recognition rate increases with respect to the base NN. This observation, however, is not true if we consider each specific reliability estimator. For each estimator, in fact, there is at least a dataset where performance degrades.

In order to analyze how the performance of the NN classifier varies with the size of the training set when self-training with the different method for reliability estimation is adopted, we repeated the previous experiment by varying the size of the training set. The results, reported in Figure 2, are expressed in terms of the error rate and refer to the performance obtained using the BEST (left column) and the END (right column) criterion respectively.

As it can be expected, the error rates curves in Figure 2 decrease as the size of the training set increases. This behavior is still more evident for the $\epsilon$-SVR (see Figures 2, $\mathrm{f}$ and $2 \mathrm{~h}$ ) that benefits from the availability of more training samples to dramatically reduce its error rate. Another interesting element of discussion derives from the comparative analysis between the curves relative to the use of the BEST and the END criteria. In fact, at least for $\epsilon$-SVR, $\psi_{A}$ and $\psi_{m e d}$, in all cases the END criterion allows to perform as good as the BEST one. This can be explained by considering that the self-training procedure terminates after only few iterations (two or three).

\section{Conclusions}

In this paper we have assessed the impact of reliability evaluation on a semisupervised learning approach, namely the self-training. The experimental analysis has been carried out on four publicly available datasets and was aimed at assessing the performance of five different reliability estimators. Even if the 
obtained results seem to confirm that self-training always (at least for the considered datasets) allows to increase the recognition rate with respect to the use of the base classifier, on the other side there is no reliability estimator that appears definitively the best. However, the behavior of the various methods when varying the size of the training dataset suggests that a simple definition of the reliability calculated using only the classifier output, as $\psi_{A}$ or $\psi_{m e d}$, can be more adequate, while a trainable reliability estimator, as $\epsilon$-SVR, can be preferred when the training set size tends to increase. The latter consideration seems to be particularly true when the number of classes is quite limited.

In this paper the robustness of the considered reliability estimators with respect to the reliability threshold $\tau$ has not been studied. It will be matter of further analysis in the future.

\section{References}

1. Blake, C., Keogh, E., Merz, C.J.: UCI Repository of machine learning databases. University of California, Department of Information and Computer Science, Irvine, CA, Irvine, CA (1998), http://www.ics.uci.edu/ mlearn/MLRepository.html

2. Chapelle, O., Zien, A., Schölkopf, B. (eds.): Semi-supervised learning. MIT Press, Cambridge (2006)

3. Cordella, L.P., Foggia, P., Sansone, C., Tortorella, F., Vento, M.: Reliability Parameters to Improve Combination Strategies in Multi-Expert Systems. Pattern Analysis and Applications 2(3), 205-214 (1999)

4. Didaci, L., Roli, F.: Using Co-training and Self-training in Semi-supervised Multiple Classifier Systems. In: Yeung, D.-Y., Kwok, J.T., Fred, A., Roli, F., de Ridder, D. (eds.) SSPR 2006 and SPR 2006. LNCS, vol. 4109, pp. 522-530. Springer, Heidelberg (2006)

5. Foggia, P., Percannella, G., Sansone, C., Vento, M.: Evaluating Classification Reliability for Combining Classifiers. In: Proceedings of the 14th International Conference on Image Analysis and Processing, Modena (IT), September 10-14, pp. 711-716 (2007)

6. Gargiulo, F., Mazzariello, C., Sansone, C.: A Self-training Approach for Automatically Labeling IP Traffic Traces. In: Kurzynski, M., Puchala, E., Wozniak, M., Zolnierek, A. (eds.) Computer Recognition Systems, vol. 2, pp. 705-717. Springer, Heidelberg (2008)

7. Giacinto, G., Roli, F.: Design of effective neural network ensembles for image classification purposes. Image Vision Computing 19(9-10), 699-707 (2001)

8. Woods, K., Kegelmeyer, W.P., Bowyer, K.W.: Combination of Multiple Classifiers Using Local Accuracy Estimates. IEEE Trans. Pattern Anal. Mach. Intell. 19(4), 405-410 (1997)

9. Kuncheva, L.I., Whitaker, C.J., Narasimhamurthy, A.: A case-study on naive labelling for the nearest mean and the linear discriminant classifiers. Pattern Recognition 41, 3010-3020 (2008)

10. Rosenberg, C., Hebert, M., Schneiderman, H.: Semi-supervised self-training of object detection models. In: Proceedings of the Seventh IEEE Workshop on Applications of Computer Vision, Breckenridge, USA, January 5-7 (2005)

11. Zhu, X.: Semi-supervised learning literature survey. Technical Report 1530, Computer Sciences, University of Wisconsin-Madison (2006) 